\title{
Plane sudden expansion flows of viscoelastic liquids
}

\author{
R.J. Poole ${ }^{\mathrm{a}, *}$, M.A. Alves ${ }^{\mathrm{b}}$, P.J. Oliveira ${ }^{\mathrm{c}}$, F.T. Pinho ${ }^{\mathrm{d}, \mathrm{e}}$

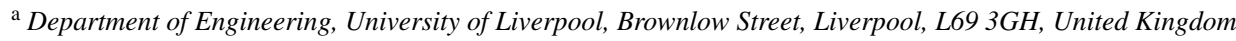 \\ ${ }^{\mathrm{b}}$ Departamento de Engenharia Química, CEFT, Faculdade de Engenharia, Universidade do Porto, Rua Dr. Roberto Frias, 4200-465 Porto, Portugal \\ ${ }^{\mathrm{c}}$ Departamento de Engenharia Electromecânica, Unidade Materiais Têxteis e Papeleiros, Universidade da Beira Interior, 6201-001 Covilhã, Portugal \\ ${ }^{\mathrm{d}}$ CEFT, Faculdade de Engenharia, Universidade do Porto, Rua Dr. Roberto Frias, 4200-465 Porto, Portugal \\ e Universidade do Minho, Largo do Paço, 4704-553 Braga, Portugal
}

Received 24 June 2006; received in revised form 15 September 2006; accepted 8 November 2006

\begin{abstract}
We report a systematic numerical investigation of the creeping flow of three different viscoelastic models, the UCM, Oldroyd-B and the linear form of the PTT model, through a 1:3 planar sudden expansion. Although the effect of elasticity is to reduce both the length and intensity of the recirculation region downstream of the expansion, we show that this reduction is much lower than previous studies have suggested and that, at high Deborah number, a significant region of recirculation still exists for all of the models studied.
\end{abstract}

(C) 2006 Elsevier B.V. All rights reserved.

Keywords: Sudden expansion; Creeping flow; UCM; Oldroyd-B; PTT

\section{Introduction}

In stark contrast to the flow through sudden contractions, the laminar flow of viscoelastic liquids through sudden expansions has received very little attention in the literature and is largely restricted to a handful of papers. Perhaps the first to investigate these types of flows were Halmos and Boger [1] who conducted experiments in axisymmetric geometries. Streak photography was used for flow visualisation while a flash technique was used to obtain centreline velocity estimates. In earlier work, Halmos et al. [2,3], these authors presented a numerical solution with experimental validation for the flow of inelastic, power-law fluids through an axisymmetric sudden expansion. Halmos and Boger concluded that as a viscoelastic fluid flows through a sudden expansion it releases some of its stored energy, resulting in an expansion of the main flow and compression of the "secondary cell" (the recirculation region).

Darwish et al. [4] and later Missirlis et al. [5] used a finitevolume technique to simulate the flow of a viscoelastic liquid through a 1:4 plane sudden expansion using the upper convected Maxwell (UCM) model. Missirlis et al. conducted simulations, at essentially creeping-flow conditions $(R e=0.1)$, up to a Debo-

\footnotetext{
* Corresponding author. Tel.: +44 1517944806; fax: +44 1517944848.

E-mail address: robpoole@liv.ac.uk (R.J. Poole).
}

rah number $(D e)$ of three. They showed that the suppression of vortex activity is directly related to $D e$ and concluded that recirculation is completely eliminated for $D e>3$. However, these conclusions must be viewed with caution because the meshes used by Missirlis et al. were, as a consequence of the computing power available at the time, rather coarse. The effects of such coarse meshes are significant: comparison of their estimation of the reattachment length for the Newtonian fluid flow shows a discrepancy of $25 \%$ compared to recent calculations [6]. In addition to this mesh coarseness the Upwind Differencing Scheme (UDS) was used for the viscoelastic stress terms in the constitutive equation and it is well known that this scheme leads to excessive numerical diffusion and its use is discouraged $[7,8]$.

Townsend and Walters [9] also used flow visualisation to observe the flowfield downstream of both a two-dimensional (expansion ratio 3:40) and a three-dimensional expansion (expansion ratio 3:3:40) for aqueous solutions of a polyacrylamide $(0.15 \%)$, a xanthan gum $(0.1 \%)$ and a glass fibre suspension $(0.025 \%)$. The conclusion drawn from their study was that the viscoelasticity of the polymer solutions damped out the vortex activity and caused any recirculating fluid to be pushed into the corners of the expansion. They used the 'linear' form of the Phan-Thien and Tanner (PTT) model [10] in an attempt to simulate the viscoelastic behaviour of the polyacrylamide solution. The PTT simulation $\left(\varepsilon=0.02, \beta=1 / 9, R e=\rho U L / \eta_{0}=10\right.$ and $\mathrm{We}=\lambda U / L=1$, where $U$ and $L$ are characteristic velocity 
and length scales, $\eta_{0}$ is the sum of the solvent and polymer viscosity, $\beta$ is the ratio of solvent to total viscosity and $\lambda$ is the relaxation time) produced results in good qualitative agreement with the flow visualisation.

The experiments of Townsend and Walters [9] were also used as the basis for comparison in the numerical simulation work of Baloch et al. [11] who modeled expansion flows in two (expansion ratios of 3:40 and 1:80) and three dimensions (expansion ratio 3:3:40), again using the linear form of the PTT model. Once again good qualitative agreement with the experimental visualisations was found and the conclusion again drawn that viscoelasticity suppresses vortex activity and that this suppression is linked to the phenomenon of 'extrudate-swell'.

More recently, Oliveira [12] investigated viscoelastic effects in the flow of a viscoelastic liquid through a 1:3 sudden expansion at higher Reynolds numbers $(>10)$ where for Newtonian fluid flow, asymmetry of the downstream flowfield is known to occur [13-15]. A modified FENE-CR constitutive equation [16] was employed and it was observed that in this relatively high-Re regime the effect of viscoelasticity was a stabilising one, i.e. the switch from symmetric to asymmetric flow occurred at higher $R e$ than the corresponding Newtonian case.

The general view from the limited existing literature regarding viscoelastic expansion flow at low Reynolds number is that the amount of recirculating fluid seems to be suppressed and then, at high enough $D e$, totally eliminated. The mechanism for this suppression is thought to be the ability of the polymer molecules to relax their stresses upon entry to the expansion and, in general terms, similar to the phenomena of 'extrudate-swell'. In this paper we report the results of a systematic numerical investigation, using a finite volume technique, of the creeping flow $(R e=0.01)$ of three 'model' viscoelastic fluids, the UCM, Oldroyd-B and the PTT models, through a 1:3 two-dimensional sudden expansion using meshes an order of magnitude or-more refined than the most recent previous study [5]. Our results show that the degree to which recirculation is in fact suppressed is far less than previous studies have suggested and that, contrary to the early works, at high Deborah number a significant recirculation region still exists downstream of the expansion for these models.

\section{Governing equations and numerical method}

The flow is assumed to be laminar and incompressible, and the fluid a mixture of a polymer in a Newtonian solvent. The governing equations are then those expressing conservation of mass:

$\nabla \cdot \boldsymbol{u}=0$

and momentum:

$\rho\left[\frac{\partial \boldsymbol{u}}{\partial t}+\nabla \cdot \boldsymbol{u} \boldsymbol{u}\right]=-\nabla p+\eta_{\mathrm{s}} \nabla \cdot \nabla \boldsymbol{u}+\nabla \cdot \boldsymbol{\tau}$

together with an appropriate constitutive relation for the extra polymer contribution to the stress tensor $\tau$. One of the most widely applicable viscoelastic models is a simplified form of the PTT model due to Phan-Thien and Tanner [10]:

$$
\begin{aligned}
\lambda\left[\frac{\partial \boldsymbol{\tau}}{\partial T}+\nabla \cdot \boldsymbol{u} \boldsymbol{\tau}\right]+f(\operatorname{Tr} \boldsymbol{\tau}) \boldsymbol{\tau}= & \eta_{\mathrm{p}}\left(\nabla \boldsymbol{u}+\nabla \boldsymbol{u}^{\mathrm{T}}\right) \\
& +\lambda\left(\boldsymbol{\tau} \cdot \nabla \boldsymbol{u}+\nabla \boldsymbol{u}^{\mathrm{T}} \cdot \boldsymbol{\tau}\right)
\end{aligned}
$$

This last Eq. (3) retains only the upper convected part of the full Gordon-Schowalter derivative. In the current study, the stress function $f(\operatorname{Tr} \boldsymbol{\tau})$ takes the linear form proposed in the original work of Phan-Thien and Tanner [10]:

$f(\operatorname{Tr} \boldsymbol{\tau})=1+\frac{\lambda \varepsilon}{\eta_{\mathrm{p}}} \operatorname{Tr}(\boldsymbol{\tau})$

In Eqs. (2)-(4) the constant model parameters are the relaxation time of the polymer $\lambda$, the zero-shear polymer viscosity $\eta_{\mathrm{p}}$, the solvent viscosity $\eta_{\mathrm{s}}$, and the extensibility parameter $\varepsilon$. Setting some of these model parameters to zero allows the well-known upper convected Maxwell (i.e. $\eta_{\mathrm{s}}$ and $\varepsilon=0$ ) and the Oldroyd-B $(\varepsilon=0)$ models to be regained [17]. For both the Oldroyd-B and PTT models, the viscosity ratio $\beta$, defined as the ratio of solvent viscosity to total viscosity, was kept constant at $\beta=1 / 9$ and the value assigned to the extensional parameter of the PTT model was $\varepsilon=0.25$, which is typical of concentrated polymer solutions or polymer melts [18]. Limited results, at a single Deborah number $(=1)$, are reported for different values of $\varepsilon$ to highlight its effect.

A fully-implicit finite-volume numerical method is used to solve Eqs. (1)-(3). The method is described in detail in [19,20] and [6] so only a brief account is given here. The method is based on a time marching pressure-correction algorithm formulated with the collocated variable arrangement. The governing equations are integrated in space over the control volumes (cells with volume $V_{\mathrm{p}}$ ) forming the computational mesh, and in time over a time step $(\Delta t)$, so that sets of linearised algebraic equations are obtained, having the general form:

$a_{P}^{\phi} \phi_{P}=\sum_{F=1}^{6} a_{F}^{\phi} \phi_{F}+S^{\phi} \Leftrightarrow\left[a^{\phi}\right]\{\phi\}=\left\{S^{\phi}\right\}$

to be solved as a matrix equation for the velocity components $(\phi=u, v)$ or the extra stress components $\left(\phi=\tau_{x x}, \tau_{y y}, \tau_{x y}\right)$. In these equations $a_{F}^{\phi}$ are coefficients accounting for convection and diffusion influences, $S^{\phi}$ are source terms encompassing all contributions not included in the coefficients, the subscript $P$ denotes the cell under consideration and subscript $F$ its corresponding neighbouring cells. The central coefficient of the discretised equations, $a_{P}^{\phi}$, is generally given by the sum of surrounding coefficients and a contribution from the timedependent terms in the original equations Eqs. (2) and (3), e.g. for $(\phi=u, v)$ :

$a_{P}=\frac{\rho V_{P}}{\Delta t}+\sum_{F=1}^{6} a_{F}$

but for the PTT stress equations $(\phi=\tau)$ an additional term is included, resulting from the $f(\operatorname{Tr} \tau)$ term in Eq. (3), which tends 


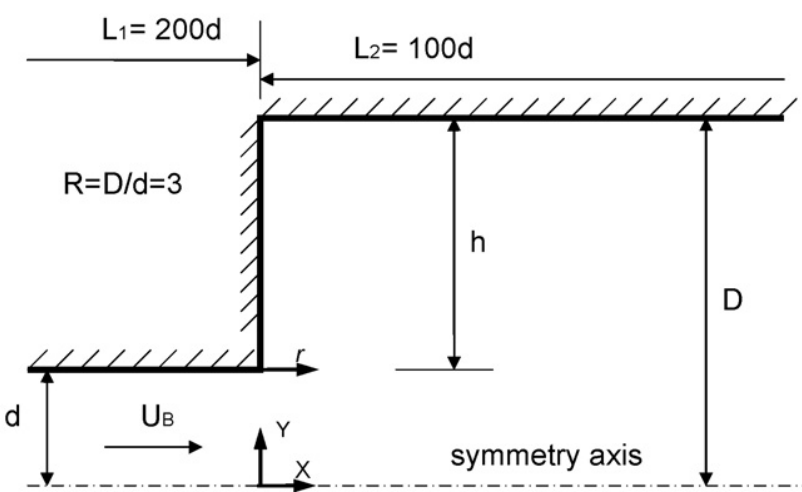

Fig. 1. Schematic of expansion geometry.

to promote stability by increasing the $a_{\mathrm{P}}$ coefficient:

$a_{P}^{\tau}=\frac{\lambda V_{P}}{\Delta t}+V_{P}\left(1+\frac{\lambda \varepsilon}{\eta_{P}} \operatorname{Tr}\left(\tau_{P}\right)\right)+\sum_{F=1}^{6} a_{F}^{\tau}$

Having assembled the coefficients and source terms, the linear sets of equations (RHS of Eq. (5)) are first solved for the extra stress components $\left(\phi=\tau_{x x}, \tau_{y y}, \tau_{x y}\right)$ then the momentum equations are solved implicitly for the two Cartesian velocity components $\phi=u$ and $v$. These newly-computed velocity components do not, in general, satisfy the continuity equation (i.e. Eq. (1)) and need therefore to be corrected by an adjustment of the pressure differences which drive them. This is accomplished by means of a pressure-correction field obtained from a pressure Poisson equation, derived from the discretised equivalent of Eq. (1) and a simplified form of Eq. (5), which is then solved with a symmetric conjugate gradient method.

Most importantly from the standpoint of accuracy is the representation of the convective terms in the constitutive equations. As the upwind differencing scheme leads to numerical diffusion we use the CUBISTA convective scheme especially designed for differential constitutive relations (proposed in [21]). This scheme has the advantage over more classical schemes (e.g. the SMART scheme of Gaskell and Lau [22]) of promoting iterative convergence when employed in conjunction with implicit methods.

\section{Geometry and computational meshes}

A schematic of the expansion geometry is shown in Fig. 1. Only half of the two-dimensional domain is used for the computations, with symmetry imposed on the centreline. For the creeping flow conditions under investigation here we believe this is a reasonable modelling assumption as bifurcations to asymmetric flow have only been observed at much higher Reynolds numbers [12]. For consistency with previous studies, the inlet channel half-width $d$ is taken as the characteristic length scale and the average velocity in the upstream channel $U_{\mathrm{B}}$ as the characteristic velocity scale. At inlet we apply uniform velocity $\left(=U_{\mathrm{B}}\right)$ and stress $(=0)$ boundary conditions and allow 200 inlet channel half-widths for the flow to fully-develop (we always checked to confirm that this length was sufficient for fully-
Table 1

Characteristics of the computational meshes

\begin{tabular}{lrrl}
\hline Mesh & NC & DOF & $\frac{\Delta x_{\min }}{d}=\frac{\Delta y_{\min }}{d}$ \\
\hline M0 & 2400 & 14400 & 0.052 \\
M0A & 2400 & 14400 & 0.026 \\
M1 & 9000 & 54000 & 0.025 \\
M1A & 9000 & 54000 & 0.0125 \\
M2 & 36000 & 216000 & 0.0125 \\
M2A & 36000 & 216000 & 0.00625 \\
M3 & 144000 & 864000 & 0.00625 \\
M3A & 144000 & 864000 & 0.003125 \\
\hline
\end{tabular}

developed flow to occur prior to the expansion plane). Stresses and pressure are, in general, normalised with $\eta_{0} U_{\mathrm{B}} / d$. For the PTT model results different normalisations are also utilized in an attempt to remove the influence of shear thinning from the data: where this occurs it is commented upon in the text. The mesh data is provided in Table 1 and comprises; the total number of control volumes in the meshes (NC), the degrees of freedom (DOF) and the minimum mesh spacing normalised with the inlet channel half-width $d$. The M2 and M2A meshes are both concentrated near the expansion plane but the expansion factor of $\mathrm{M} 2 \mathrm{~A}$ is larger and therefore the smallest CVs adjacent to the corner are smaller in dimension.

A large number of computations have been performed, over 100 in all, on the meshes defined in Table 1, for increasing values of the Deborah number $\left(D e=\lambda U_{\mathrm{B}} / d\right)$ at a constant Reynolds number corresponding to creeping flow conditions $\left(R e=2 \rho U_{\mathrm{B}} d / \eta_{0}=0.01\right)$. The majority of results reported in this paper were obtained using meshes M2 and M2A. For the UCM and Oldroyd-B simulations, where the maximum obtainable Deborah number is of order one, Mesh 2 was found to be sufficient to obtain grid-independent results. The PTT simulations in contrast, where Deborah numbers of order 100 could be obtained, were found to vary significantly $(>1 \%)$ between meshes 1 and 2 above $D e \approx 2$. The PTT simulations were therefore repeated on different meshes (denoted by the ' $A$ ' suffix) which had the same number of cells but in which the minimum cell spacing was approximately halved. Even using this methodology, as Section 6 makes clear, the PTT results still exhibited a marked grid dependency at high $D e(>10)$. Limited simulations were run using meshes M3 and M3A to estimate the numerical uncertainty of the results.

We present results of recirculation length $\left(X_{\mathrm{R}}\right.$, normalised with the half-width of the inlet channel i.e. $d$ ), recirculation intensity $\left(\Psi_{\mathrm{R}}\right.$, normalised with the inlet flow rate for the half channel i.e. between the centreline and the wall) and the pressure drop across the expansion expressed as a Couette correction $\left(\mathrm{C} \equiv\left(\Delta P-\Delta P_{f d}\right) / 2 \tau_{\mathrm{w}}\right.$, where $\tau_{\mathrm{w}}$ is the wall shear stress for the fluid in question (encompassing both the solvent and polymer contributions for the Oldroyd-B and PTT models) evaluated under fully-developed conditions in the inlet channel). In addition streamline plots, contour plots of polymer stresses together with profiles of velocity and polymer-stress in areas of interest (along the centreline and along a streamwise line from the expansion corner) are shown to illustrate the effect of elasticity on the flow field. 
Table 2

Effect of mesh refinement upon recirculation length

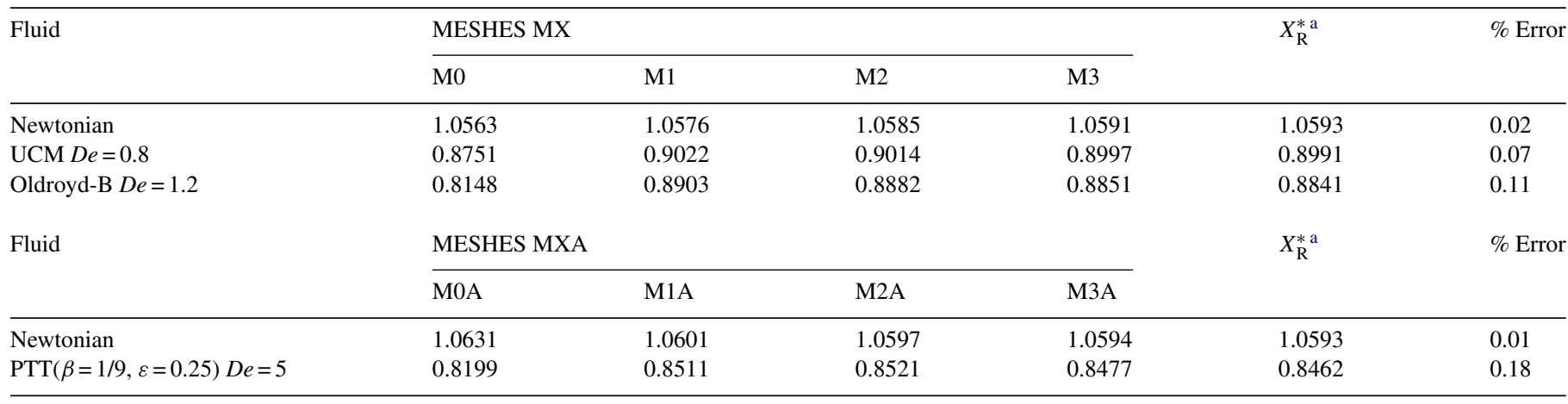

${ }^{a}$ Denotes extrapolated value using Richardson's technique.

Results are first presented for the constant shear viscosity UCM and Oldroyd-B models (Section 5), and then finally the linear form of the PTT model (Section 6) which, for the chosen parameters, exhibits a shear-thinning shear-viscosity.

\section{Grid dependency studies and numerical accuracy}

The variation of the length of recirculation with grid refinement, for the two sets of successively refined meshes MX and MXA, is provided in tabulated form in Table 2 and in graphical form in Fig. 2(a) and (b). The '\% error' given in Table 2 is a quantification of the relative difference between the predictions
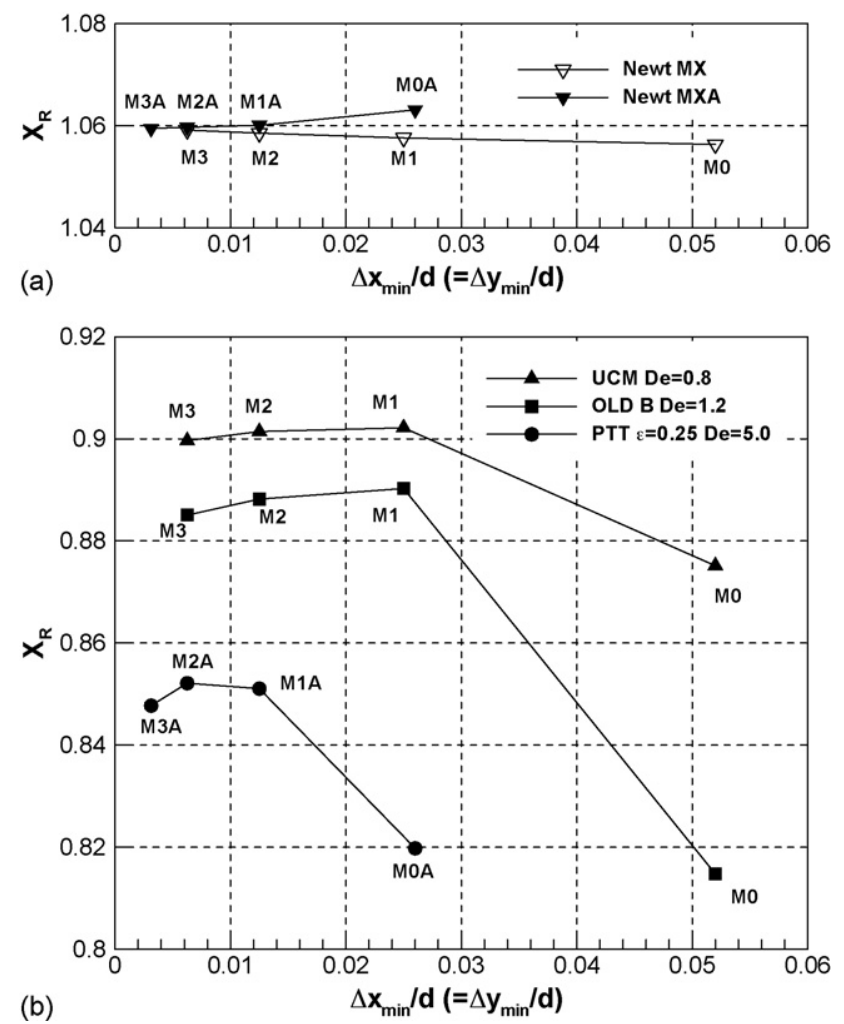

Fig. 2. Variation of recirculation length with mesh refinement: (a) Newtonian simulations (Meshes M0, M1, M2, M3, M0A, M1A, M2A and M3A); (b) viscoelastic simulations (UCM and Oldroyd-B M0, M1, M2 and M3, PTT M0A, M1A, M2A and M3A). of $X_{\mathrm{R}}$ on the finest mesh (either M3 or M3A) and the extrapolated results obtained from Richardson's extrapolation technique (denoted by $X_{\mathrm{R}}^{*}$ in Table 2). In effect, they are a measure of the uncertainty of our results. Due to the excessive time required to run simulations on the finest meshes, we only conducted these simulations for the (approximately) maximum $D e$ that could be achieved for each model in order to quantify the uncertainty in our results. As can be seen, particularly for the Newtonian simulations, the differences with grid refinement are exceeding small. The agreement between the two 'sets' of meshes (i.e. MX and MXA) for the Newtonian simulations is very good and, as they should, the extrapolated recirculation lengths agree (to 4 decimal places at least). It is interesting to note (see Fig. 2(a)) that although the paths for convergence differ on meshes MX and MXA, on account of the different cell-size expansion ratios, they eventually lead to the same converged result, a reassuring outcome. For the UCM and Oldroyd-B simulations the uncertainty increases slightly with increasing elasticity but the differences are still very small $(\sim 0.1 \%)$. The PTT simulations exhibited greater uncertainty and, on the finest mesh, we could only obtain converged solutions up to $D e \approx 5$; below this value the uncer-

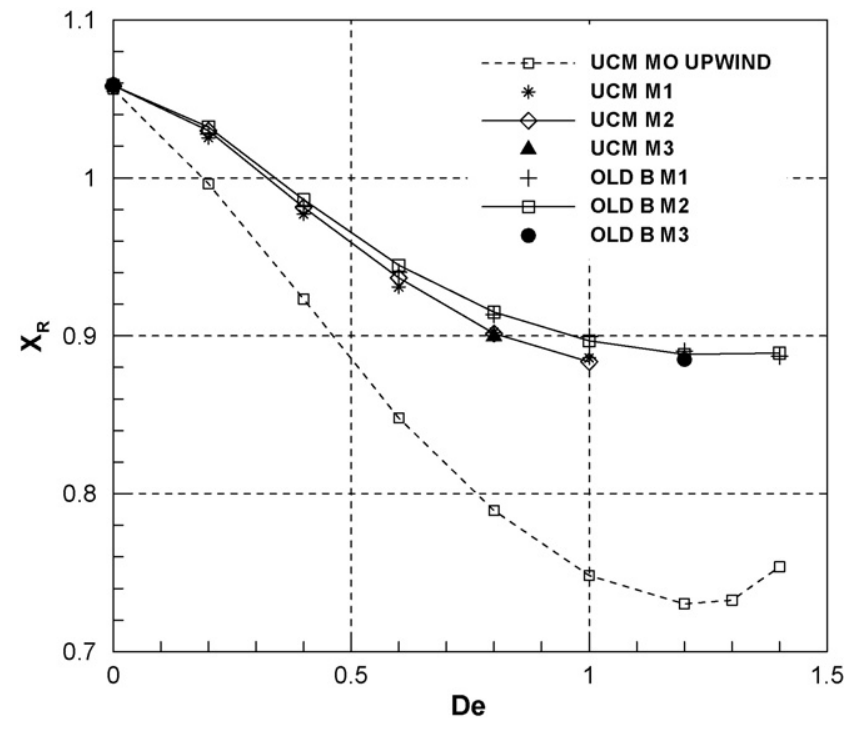

Fig. 3. Variation of recirculation length $X_{\mathrm{R}}$ with Deborah number for the UCM and Oldroyd-B models. 


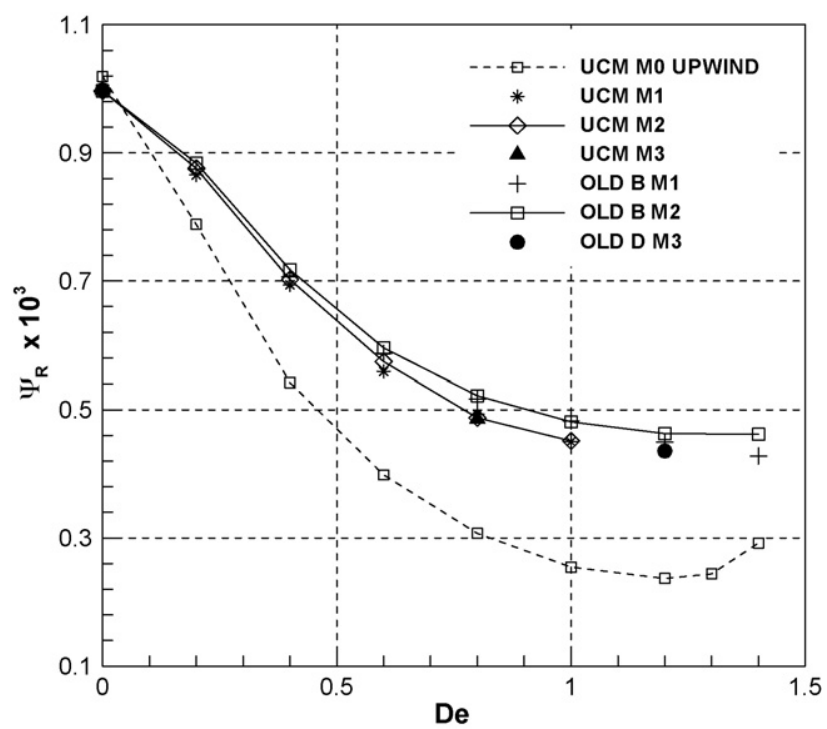

Fig. 4. Variation of recirculation intensity $\Psi_{\mathrm{R}}$ with Deborah number for the UCM and Oldroyd-B models.

tainty in our estimates of $X_{\mathrm{R}}$ is less than $0.2 \%$ but, as our later results make clear, above this level of elasticity our results exhibit greater grid dependency. Such grid dependency for the PTT model, especially at such a modest value of $\varepsilon$, is perhaps surprising and is in marked contrast to simulations in a $4: 1$ sudden contraction [6].

\section{Expansion flow of UCM and Oldroyd-B fluids}

Both the UCM and Oldroyd-B models gave very similar results and are consequently discussed together with the majority of plots for the latter model not shown for conciseness. However, with a view to providing benchmark data, tabulated results for both models are provided. The variation of the length of recirculation with increasing elasticity, as measured by the Deb-

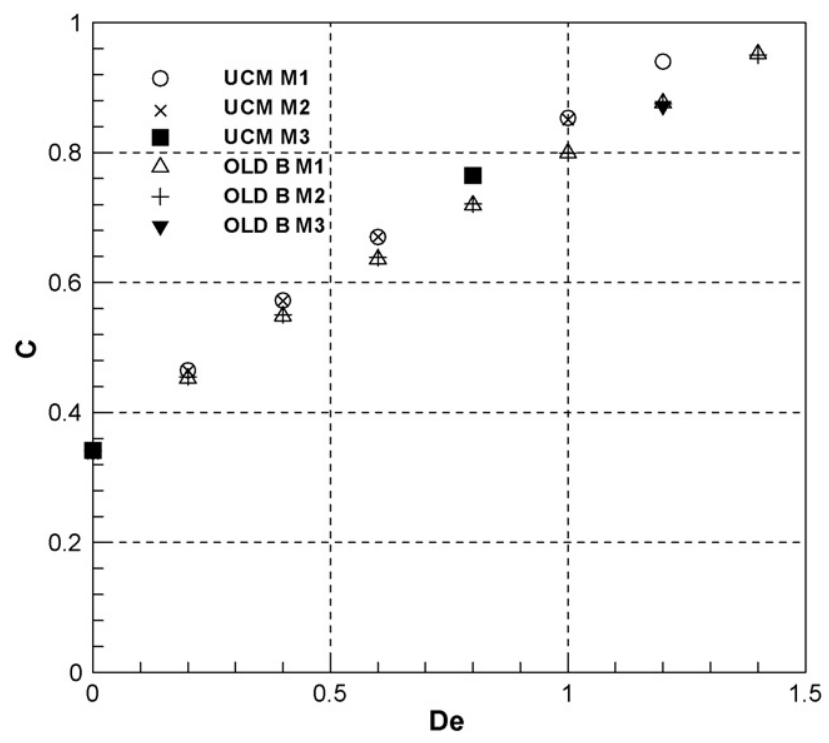

Fig. 5. Normalised extra pressure drop across expansion with increasing elasticity for the UCM and Oldroyd-B models. orah number, for the UCM and Oldroyd-B models is shown in Fig. 3. For the finest mesh convergence could not be achieved for Deborah numbers greater than 0.8 for the UCM and 1.2 for the Oldroyd-B. Despite the rather modest maximum attainable value of $D e$ the trend is clear: although increasing elasticity initially decreases the length of recirculation, at higher values of the Deborah number the length of recirculation appears to be reaching a plateau value of about 0.88 (i.e. about a $17 \%$ reduction compared to the Newtonian value). This trend is in marked contrast to the existing view of the literature, where, as we have already discussed, previous studies have shown that recirculation is continually suppressed with increasing $D e$ before being totally eliminated at some critical value of $D e$ [5]. To investigate this discrepancy we ran additional simulations on an extremely coarse mesh (M0) using the upwind differencing scheme: the results can be seen in Fig. 3 as the 'dashed' line. Although, compared to our most accurate results (i.e. meshes M2/M3 using CUBISTA), a significant reduction in $X_{\mathrm{R}}$ is observed for the viscoelastic simulations it is clear that $X_{\mathrm{R}}$ is not tending to zero with increasing $D e$. The corresponding variation of recirculation intensity with $D e$ is shown in Fig. 4. This figure shows that, in conjunction with a decrease in recirculation length with $D e$, the degree of recirculation is also reduced. In this case the percentage reduction compared to the Newtonian case is much greater (about a $60 \%$ reduction) and, once again, the decrease seems to be levelling off at higher values of $D e$. The use of a coarse mesh and the upwind scheme also severely decreases the intensity of recirculation. With the upwind scheme, the range of De could be extended up to 1.4 for the UCM model.

In Fig. 5 we plot the additional pressure drop that results from the presence of the expansion in terms of the 'Couette' correction. The Couette correction shows very little sensitivity to mesh refinement in line with previous findings for the contraction geometry [6] and increases approximately linearly with $D e$. This increase implies an enhanced pressure-drop to drive the viscoelastic fluid compared to a Newtonian fluid. Unfortunately there are no experimental measurements of pressure drop for comparison with these results. In the contraction geometry it is well-known that these, and most other viscoelastic models, predict a reduced pressure drop when, experimentally, an enhanced pressure drop is observed. Whether the same is true in the expansion geometry is open to debate. The variation of $C$ can be accurately predicted with the following correlation, based on our numerical results:

$C=0.3412+b D e+c D e^{2}$

where $b=0.6326$ and $c=-0.12085$ for the UCM model (regression factor of $R=0.99993)$. For the Oldroyd-B model a good fit is obtained with $b=0.54252$ and $c=-0.07954(R=0.999987)$. This pressure drop data is tabulated for the UCM, Oldroyd-B and PTT models in Table 3.

The streamline patterns for the UCM fluid, computed using mesh M2, are shown in Fig. 6, and are consistent with the data presented in Figs. 3 and 4 (i.e. as De increases both the length of recirculation and the intensity is reduced compared to the Newtonian case). At higher values of Deborah number $(D e>0.8)$ 
Table 3

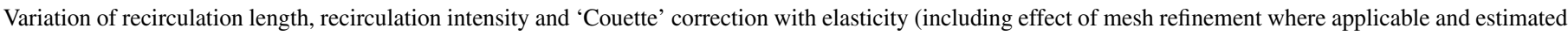
uncertainty based on Richardson extrapolation)

\begin{tabular}{|c|c|c|c|c|c|c|c|c|c|}
\hline \multicolumn{10}{|c|}{ (a) UCM model } \\
\hline \multirow[t]{2}{*}{$D e$} & \multicolumn{2}{|l|}{$X_{\mathrm{R}}$} & \multirow[t]{2}{*}{$X_{\mathrm{R}}^{* \mathrm{a}}$} & \multicolumn{2}{|c|}{$\Psi_{\mathrm{R}}\left(\mathrm{x} 10^{3}\right)$} & \multirow[t]{2}{*}{$\Psi_{\mathrm{R}}^{* \mathrm{a}}$} & \multicolumn{2}{|l|}{$C$} & \multirow[t]{2}{*}{$C^{* \mathrm{a}}$} \\
\hline & M2 & M3 & & M2 & M3 & & M2 & M3 & \\
\hline 0.0 & 1.0585 & 1.0591 & 1.0593 & 0.9967 & 0.9980 & 0.9984 & 0.3412 & 0.3416 & 0.3418 \\
\hline 0.2 & 1.0300 & & & 0.8758 & & & 0.4682 & & \\
\hline 0.4 & 0.9814 & & & 0.7022 & & & 0.5761 & & \\
\hline 0.6 & 0.9367 & & & 0.5742 & & & 0.6746 & & \\
\hline 0.8 & 0.9014 & 0.8997 & 0.8991 & 0.4874 & 0.4859 & 0.4854 & 0.7668 & 0.7652 & 0.7647 \\
\hline 1.0 & 0.8835 & & & 0.4514 & & & 0.8555 & & \\
\hline
\end{tabular}

(b) Oldroyd-B model $(\beta=1 / 9)$

\begin{tabular}{|c|c|c|c|c|c|c|c|c|c|}
\hline \multirow[t]{2}{*}{$D e$} & \multicolumn{2}{|l|}{$X_{\mathrm{R}}$} & \multirow[t]{2}{*}{$X_{\mathrm{R}}^{* \mathrm{a}}$} & \multicolumn{2}{|c|}{$\Psi_{\mathrm{R}}\left(\mathrm{x} 10^{3}\right)$} & \multirow[t]{2}{*}{$\Psi_{\mathrm{R}}^{* \mathrm{a}}$} & \multicolumn{2}{|l|}{$C$} & \multirow[t]{2}{*}{$C^{* \mathrm{a}}$} \\
\hline & M2 & M3 & & M2 & M3 & & M2 & M3 & \\
\hline 0.0 & 1.0585 & 1.0591 & 1.0593 & 0.9967 & 0.9980 & 0.9984 & 0.3412 & 0.3416 & 0.3418 \\
\hline 0.2 & 1.0322 & & & 0.8840 & & & 0.4549 & & \\
\hline 0.4 & 0.9862 & & & 0.7175 & & & 0.5506 & & \\
\hline 0.6 & 0.9447 & & & 0.5956 & & & 0.6386 & & \\
\hline 0.8 & 0.9150 & & & 0.5217 & & & 0.7208 & & \\
\hline 1.0 & 0.8968 & & & 0.4812 & & & 0.7991 & & \\
\hline 1.2 & 0.8882 & 0.8851 & 0.8841 & 0.4629 & 0.4358 & 0.4268 & 0.8752 & 0.8711 & 0.8698 \\
\hline 1.4 & 0.8891 & & & 0.4618 & & & 0.9497 & & \\
\hline
\end{tabular}

(c) PTT model $(\beta=1 / 9, \varepsilon=0.25)$

\begin{tabular}{|c|c|c|c|c|c|c|c|c|c|}
\hline \multirow[t]{2}{*}{$D e$} & \multicolumn{2}{|l|}{$X_{\mathrm{R}}$} & \multirow[t]{2}{*}{$X_{\mathrm{R}}^{* \mathrm{a}}$} & \multicolumn{2}{|c|}{$\Psi_{\mathrm{R}}\left(\mathrm{x} 10^{3}\right)$} & \multirow[t]{2}{*}{$\Psi_{\mathrm{R}}^{* \mathrm{a}}$} & \multicolumn{2}{|l|}{$C$} & \multirow[t]{2}{*}{$C^{* \mathrm{a}}$} \\
\hline & $\mathrm{M} 2 \mathrm{~A}$ & M3A & & M2A & M3A & & M2A & M3A & \\
\hline 0.0 & 1.0597 & 1.0594 & 1.0593 & 1.0005 & 0.9996 & 0.9993 & 0.3421 & 0.3419 & 0.3419 \\
\hline 0.5 & 0.9827 & & & 0.7108 & & & 0.5976 & & \\
\hline 1.0 & 0.9442 & & & 0.6137 & & & 0.7270 & & \\
\hline 2.0 & 0.9132 & & & 0.5557 & & & 0.8517 & & \\
\hline 3.0 & 0.8878 & & & 0.5099 & & & 0.9129 & & \\
\hline 4.0 & 0.8671 & & & 0.4751 & & & 0.9493 & & \\
\hline 5.0 & 0.8521 & 0.8477 & 0.8462 & 0.4510 & 0.4402 & 0.4366 & 0.9725 & 0.9719 & 0.9716 \\
\hline 6.0 & 0.8410 & & & 0.4340 & & & 0.9882 & & \\
\hline 10 & 0.8266 & & & 0.4044 & & & 1.016 & & \\
\hline 15 & 0.8320 & & & 0.3912 & & & 1.022 & & \\
\hline 20 & 0.8443 & & & 0.3844 & & & 1.018 & & \\
\hline 25 & 0.8594 & & & 0.3821 & & & 1.011 & & \\
\hline 30 & 0.8740 & & & 0.3805 & & & 1.003 & & \\
\hline 35 & 0.8881 & & & 0.3796 & & & 0.9944 & & \\
\hline 50 & 0.9260 & & & 0.3743 & & & 0.9702 & & \\
\hline 60 & 0.9453 & & & 0.3679 & & & 0.9561 & & \\
\hline 80 & 0.9772 & & & 0.3555 & & & 0.9305 & & \\
\hline 100 & 0.9966 & & & 0.3399 & & & 0.9084 & & \\
\hline
\end{tabular}

a Denotes extrapolated value using Richardson's technique.

what appears to be a small 'lip vortice' is in evidence along the wall of the inlet channel slightly upstream of the expansion plane $(x / d=0)$. With mesh refinement the length of this vortice decreases from approximately $0.08 \mathrm{~d}$ (mesh M2) to $0.06 \mathrm{~d}$ (mesh M3) and its centre moves closer to the expansion corner showing that, although small, it will remain finite with further mesh refinement. A plot of the velocity along the centreline of the expansion, shown in Fig. 7, shows that at high $D e$ a velocity overshoot is observed immediately upstream of the expansion plane, a feature also reported by Oliveira [12] with the FENECR model. This suggests that, although too small to be easily observed in the streamline plots of Fig. 6, the streamlines must be converging rather than diverging immediately upstream of the expansion. As a consequence of being far away from the sharp corner these profiles are mesh independent (i.e. this overshoot is also observed in mesh M3). A strong convergence of the streamlines at this location would be consistent with flow separation at the wall (i.e. the formation of a lip vortice) and may be the root of our convergence problems at higher Deborah number.

In Fig. 8 we plot the variation of the three non-zero stress components (i.e. $\tau_{x x}, \tau_{x y}$ and $\tau_{y y}$ ) along a streamwise line emanating from the expansion corner (shown in Fig. 1 by the line denoted 

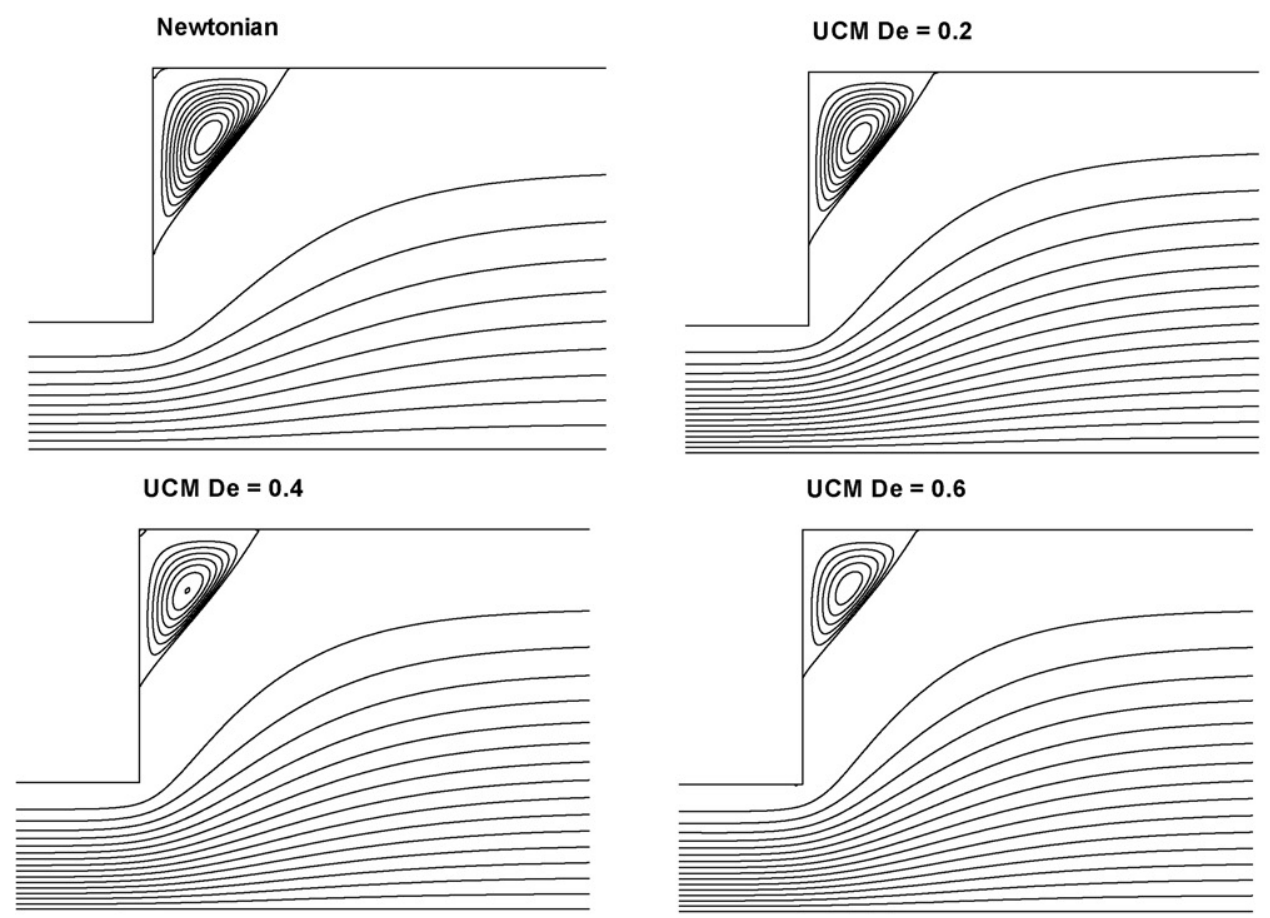

UCM De $=0.8$
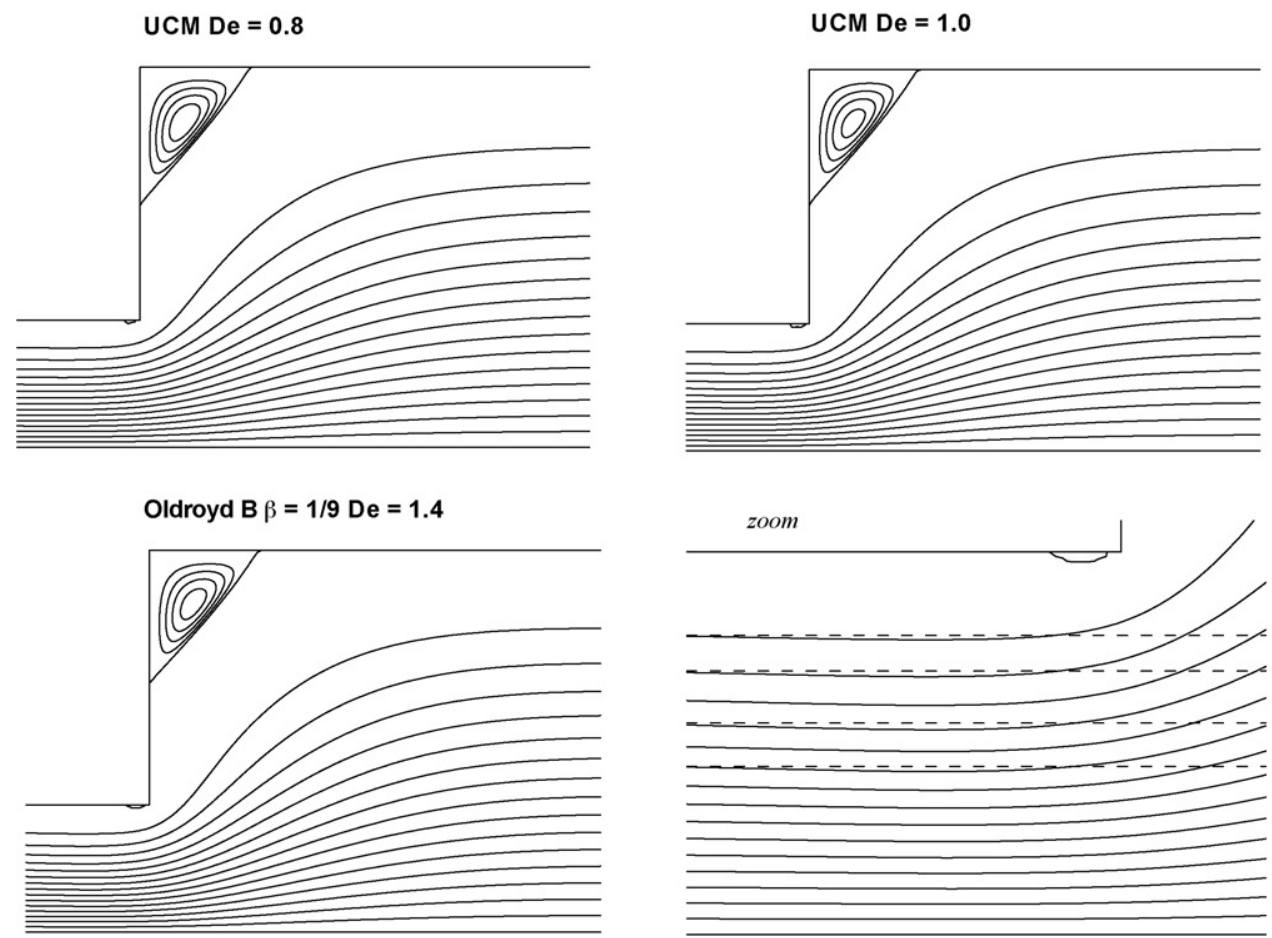

Fig. 6. Streamline patterns for the UCM and Oldroyd-B models (mesh M2). Note: $\Delta \Psi_{\mathrm{R}}=0.1 \times 10^{-3}$ in recirculation region. Zoomed section shows converging streamlines immediately upstream of expansion for Oldroyd-B $D e=1.4$ (note: horizontal, parallel dashed lines are for illustrative purposes only).

' $r$ ') for the UCM model at $D e=0.8$ on mesh M3. All three stresses exhibit the same asymptotic variation as they approach the corner, varying as $r^{-2 / 3}$ (represented as the straight lines of slope $-2 / 3$ in the $\log -\log$ plot of Fig. 8), in agreement with the theoretical predictions of Hinch [23].

Contours of shear stress and streamwise normal stresses are shown in Figs. 9 and 10, respectively. The contours for the shear and normal stresses in the expansion region show some interesting differences between the Newtonian and the viscoelastic flows. First, whereas for the Newtonian fluid the contours emanate from the corner, for the viscoelastic fluids they emanate from a wider region around the corner (upstream and downstream). Secondly, for each stress component, the contours for the various elastic fluids have more similarities between themselves than in comparison with the corresponding plot for the Newtonian fluid. The shear stress, shown in Fig. 9, exhibits a 


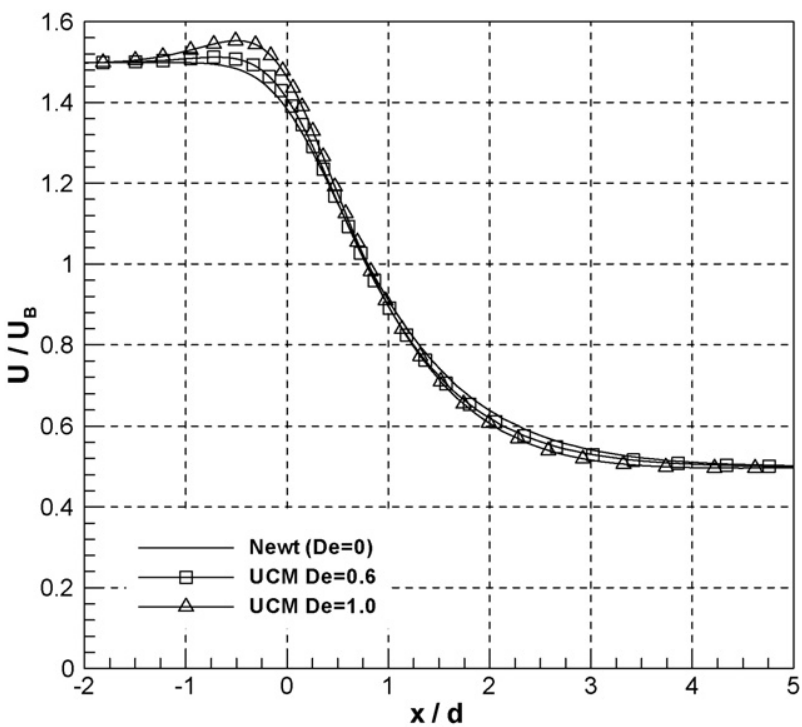

Fig. 7. Variation of streamwise velocity along centreline of expansion, close to expansion plane $(x / d=0)$, for the UCM model (Mesh M2). To avoid overcrowding only limited data $(D e=0,0.6$ and 1.0) is shown.

larger positive maximum stress region downstream of the corner for the UCM and Oldroyd-B models than for the Newtonian fluid for which this region basically coincides with the geometric singularity, followed in the two viscoelastic cases by a local stress minimum and then the stresses relax to their fully-developed values. For the PTT fluid the same events take place, but the levels of the stress and the size of these regions are distorted by shear-thinning. The flow kinematics of the PTT model are presented and discussed in full in the following section.

For the streamwise normal stress plots in Fig. 10, the situation is quite different. For the Newtonian fluid $\tau_{x x}$ is zero in the upstream and downstream fully-developed flows and in the expansion region this stress is simply proportional to $\partial U / \partial x$,

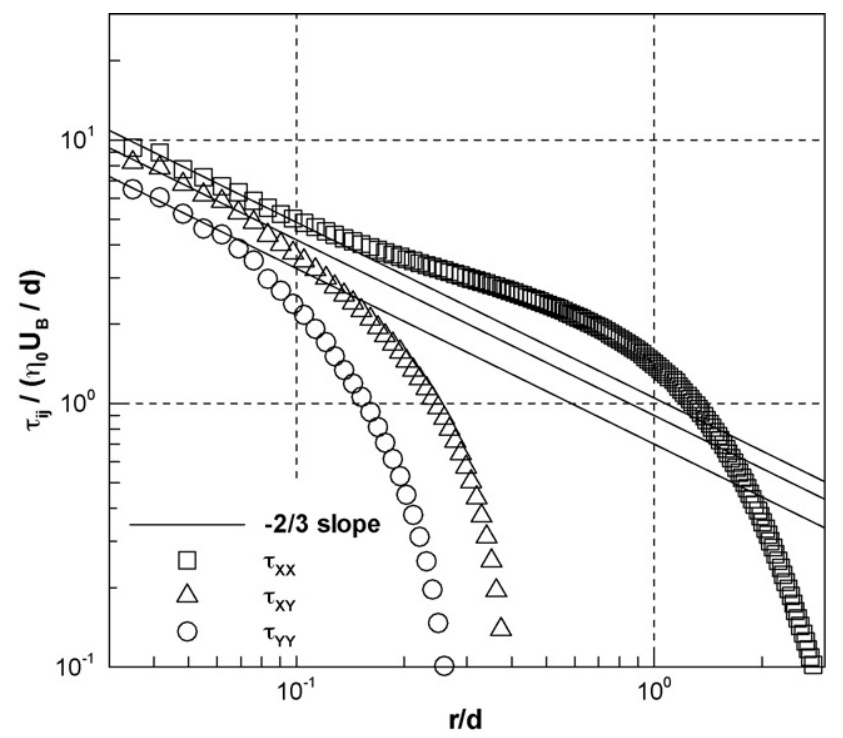

Fig. 8. Asymptotic variation of stresses along a streamwise line from expansion corner $(x, y=0, d)$ for the UCM model simulations $D e=0.8$ using mesh M3.
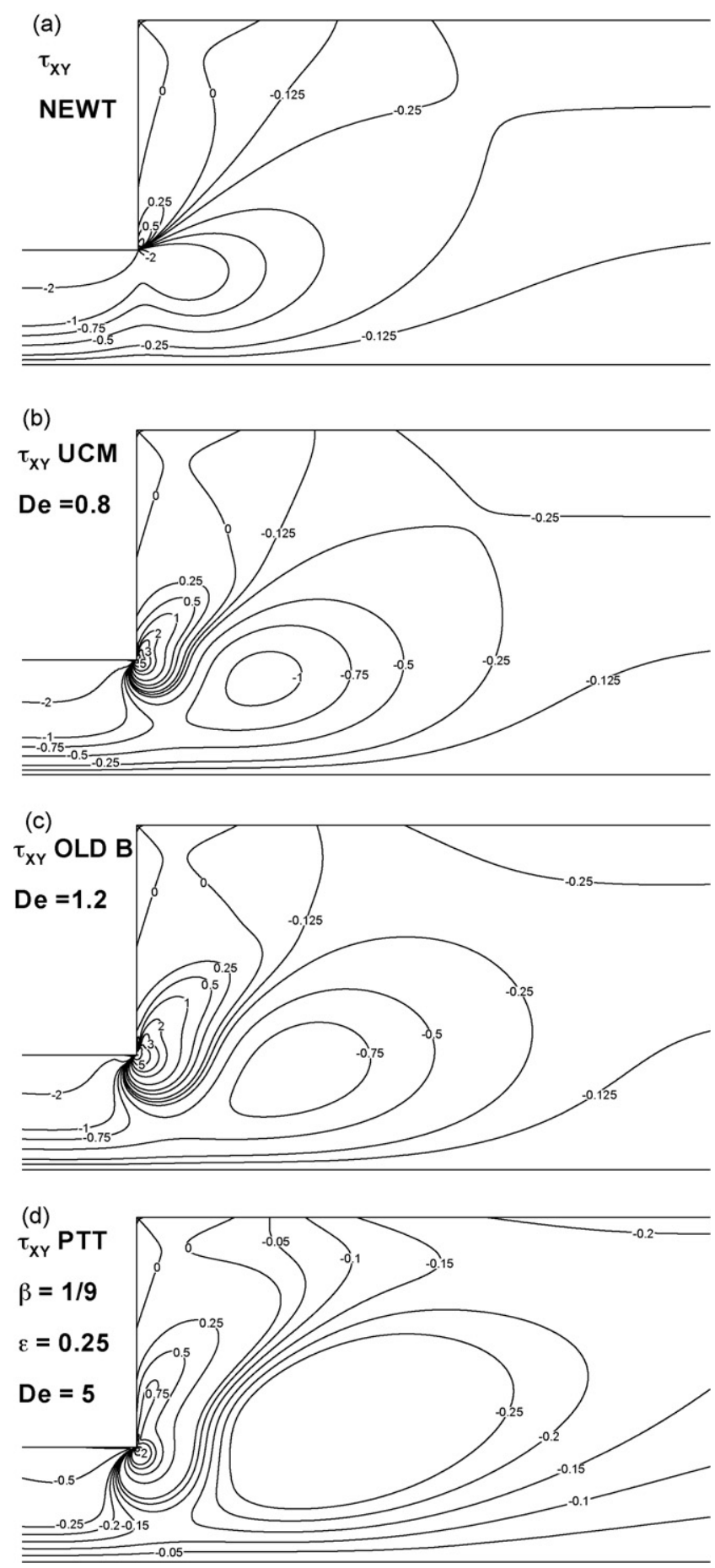

Fig. 9. Contours of normalised shear stress $\tau_{x y}$ for (a) Newtonian M3; (b) UCM $D e=0.8 \mathrm{M} 3$; (c) Oldroyd-B $D e=1.2 \mathrm{M} 3$; and (d) $\mathrm{PTT}(\beta=1 / 9, \varepsilon=0.25) D e=5.0$ M3A.

highlighting the fluid deceleration in the centreline region and the acceleration in the vicinity of the corner. The elastic nature of the other fluids is in evidence in the remaining three plots which share similarities, but are quite different from the Newtonian plot. For the PTT fluid a local positive maximum is also seen downstream of the expansion which is absent from the plots for the UCM and Oldroyd-B models. Contours of $\tau_{y y}$ not shown here, display similar behaviour to $\tau_{x x}$ along the centreline (in the 

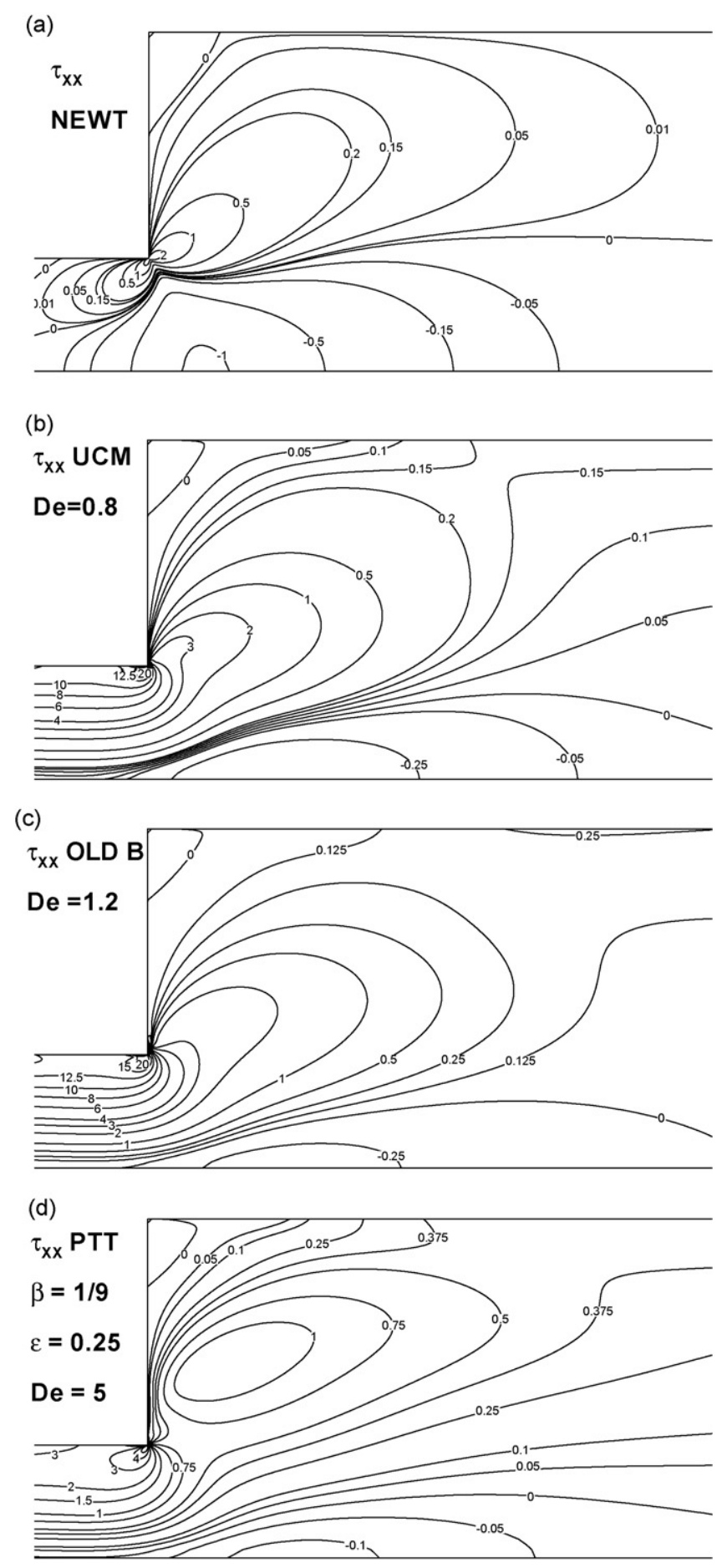

Fig. 10. Contours of normalised streamwise normal stress $\tau_{x x}$ for (a) Newtonian M3; (b) UCM $D e=0.8 \mathrm{M} 3$; (c) Oldroyd-B $D e=1.2 \mathrm{M} 3$; and (d) PTT ( $\beta=1 / 9$, $\varepsilon=0.25) D e=5.0 \mathrm{M} 3 \mathrm{~A}$

purely-extensional region of the flow) but exhibit differences in a region close to the corner, where small, closed zones of high stresses are in evidence.

For the Oldroyd-B model we were able to obtain converged solutions to slightly higher Deborah numbers $(D e=1.4 \mathrm{com}$ pared to $D e=1.0$ for UCM using mesh M2) and this higher De range allows us to confirm our findings regarding the 'plateau- ing' out of the recirculation length at high Deborah number (see Figs. 3 and 4 for example). The reduction in $X_{R}$ compared to the Newtonian value is slightly less for the Oldroyd-B model than for the UCM model but it is clear that at the highest Deborah number a significant region of recirculation still exists $\left(X_{\mathrm{R}} \approx 0.89\right.$, i.e. only a $16 \%$ reduction compared to the Newtonian). The streamline plots are very similar to those for the UCM and are not shown, except for the case $D e=1.4$ with a zoomed inset highlighting both the "lip" vortex and the converging nature of the streamlines (Fig. 6). We believe that the plateau in $X_{\mathrm{R}}$ observed at high Deborah number is a consequence of two competing elastic effects. Certainly, as previous studies have suggested, as the fluid enters the expansion its stresses are free to relax and this causes an expansion of the main flow and hence a reduction in recirculation (this may be thought of, in crude terms, as a 'confined' form of extrudate (or die) swell). However, in addition to this effect, increasing elasticity causes a convergence of the streamlines just upstream of the expansion plane (observe the 'zoomed' streamlines close to the expansion plane in Fig. 6 for $D e=1.4$ ) which has, because in essence the fluid now 'sees' a larger expansion ratio, the opposite effect.

\section{Expansion flow of PTT model fluid (linear form, $\beta=1 / 9$ and $\varepsilon=0.25$ )}

Results of recirculation length and intensity for the PTT model are shown in Figs. 11 and 12, respectively. For this model we were able, at least using M2A, to obtain converged solutions up to a $D e \approx 100$. As can be seen from inspection of Fig. 11, the variation of the recirculation length with Deborah number is considerably more complex than for the UCM and Oldroyd-B models. For De up to about 5, much as for the UCM and Oldroyd-B models, $X_{\mathrm{R}}$ is seen to decrease with increasing $D e$. This trend is seen more clearly in the inset of Fig. 11. As De increases still further the recirculation length begins to increase until by $D e=100$ it is approaching the Newtonian value (in fact it is about 6\% lower than the Newtonian value at $D e=100$ ). In contrast the intensity of recirculation is seen to decrease rapidly up until $D e=5$ before in the region where $X_{\mathrm{R}}$ is seen to increase it decreases very slowly from $0.38 \times 10^{-3}$ at $D e=10$ to about $0.34 \times 10^{-3}$ at $D e=100$. So, although the length of the recirculation region in the streamwise direction is seen to increase for $D e>10$, the intensity of recirculation is still decreasing, albeit very slowly. These effects can clearly be seen in the streamline plots of Fig. 13 as can a 'skewing' of the recirculation region, from an approximately triangular shape, for high Deborah numbers. It is this stretching of the recirculation region in the streamwise direction that results in the increases in $X_{\mathrm{R}}$ shown in Fig. 11. It is interesting to note that the 'width' of the recirculation region in the transverse direction along the sidewall initially decreases with $D e$ and then remains approximately constant for De $>10$.

The excess pressure drop across the expansion for the PTT model, again expressed as the 'Couette' correction, is shown in Fig. 14. In agreement with the UCM and Oldroyd-B models, the PTT model exhibits an enhanced pressure drop compared to the Newtonian simulations, reaching a maximum at $D e \approx 15$ 


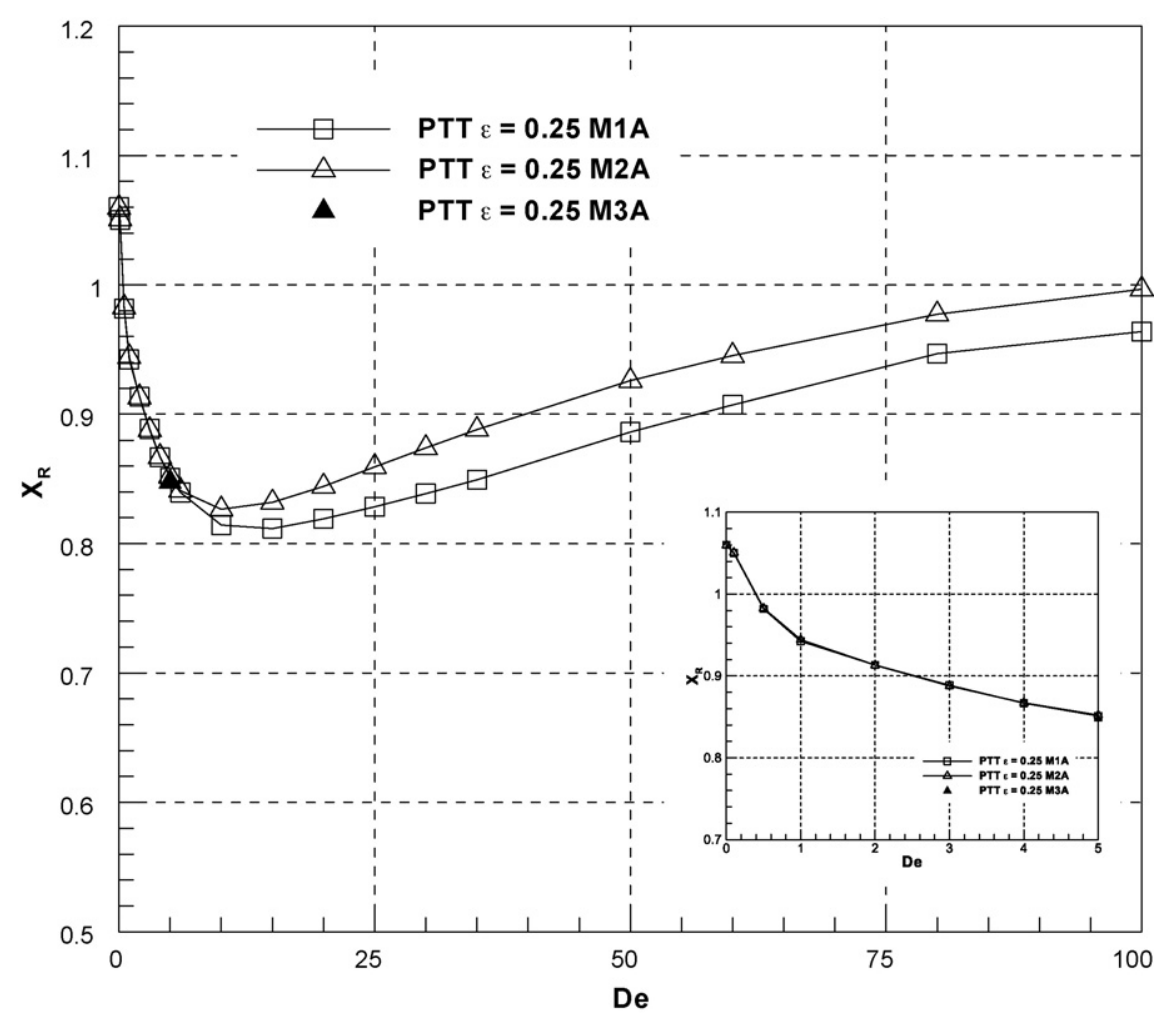

Fig. 11. Variation of recirculation length $X_{\mathrm{R}}$ with Deborah number for the PTT model $(\beta=1 / 9, \varepsilon=0.25)$

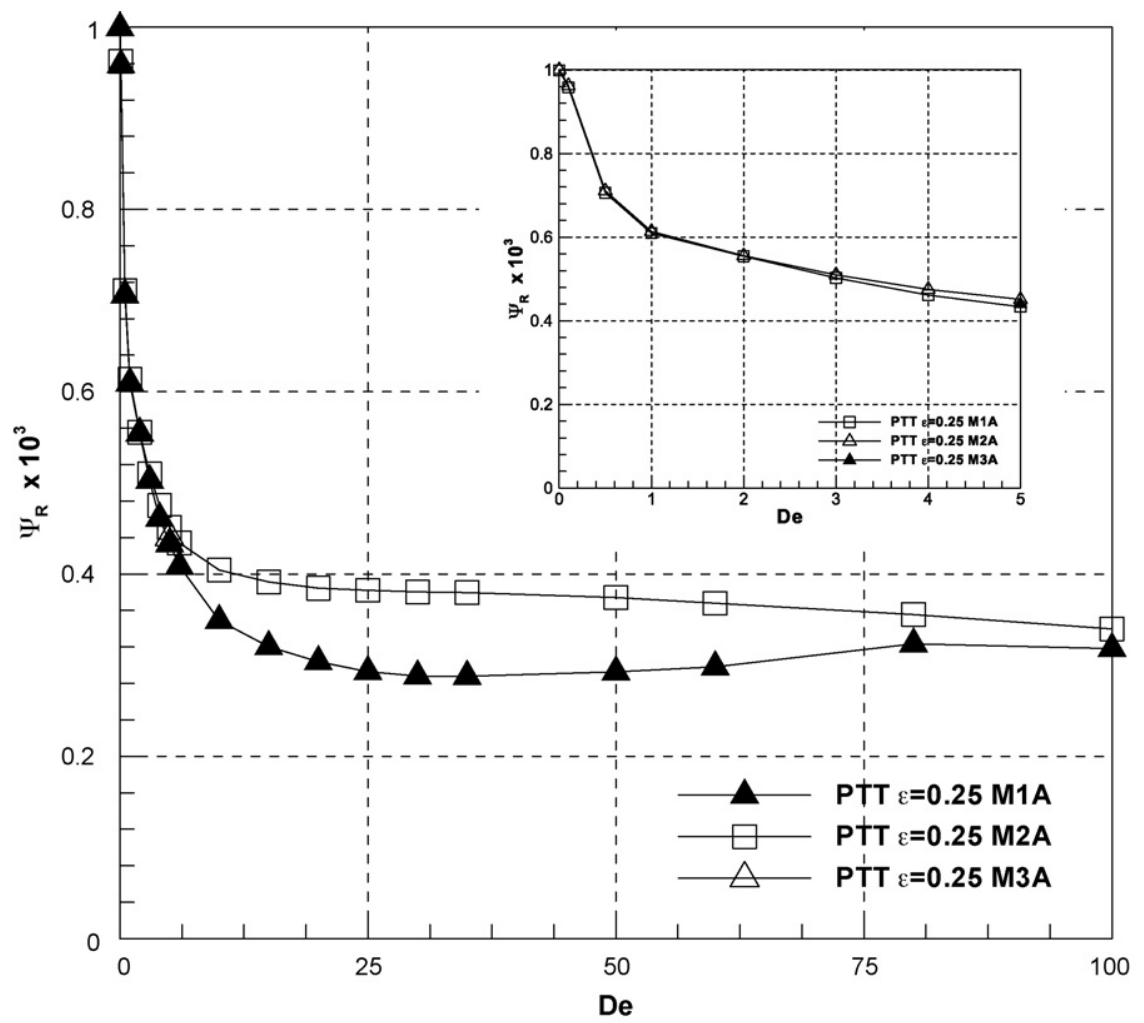

Fig. 12. Variation of recirculation intensity $\Psi_{\mathrm{R}}$ with Deborah number for the PTT model $(\beta=1 / 9, \varepsilon=0.25)$. 


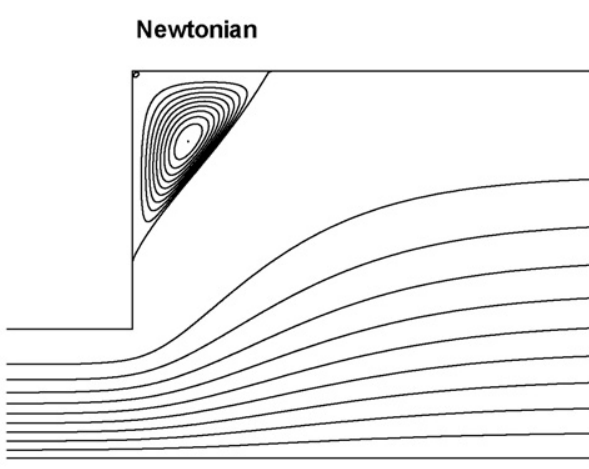

PTT $\varepsilon=0.25 \beta=1 / 9 \mathrm{De}=5.0$

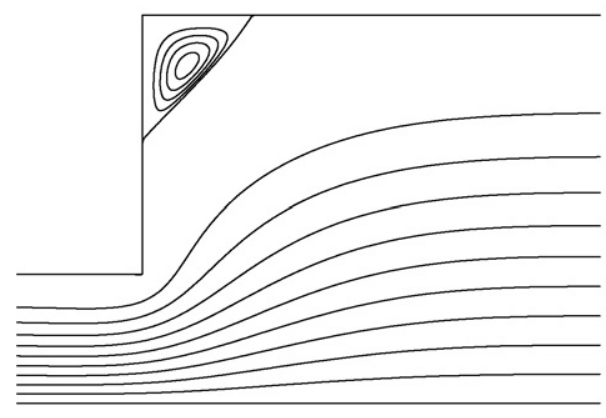

PTT $\varepsilon=0.25 \beta=1 / 9$ De $=50$

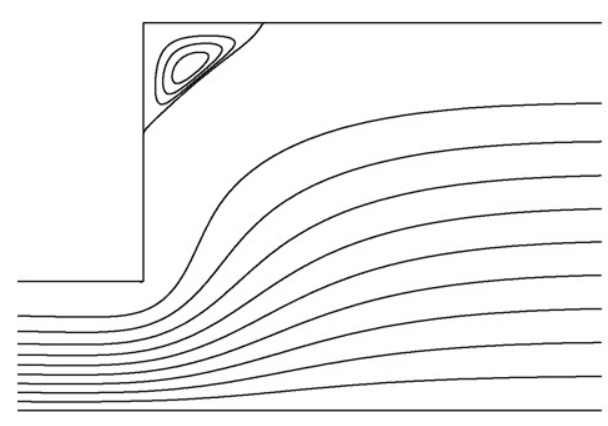

PTT $\varepsilon=0.25 \beta=1 / 9 \mathrm{De}=2.0$

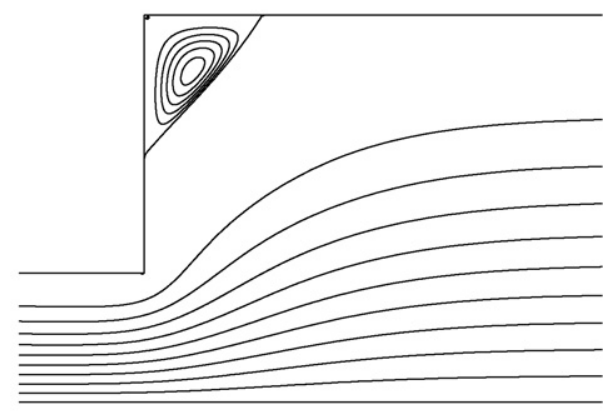

PTT $\varepsilon=0.25 \beta=1 / 9 \mathrm{De}=10.0$

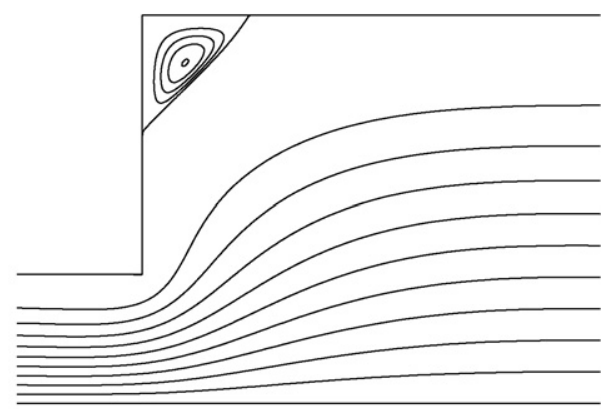

PTT $\varepsilon=0.25 \beta=1 / 9 \mathrm{De}=100$

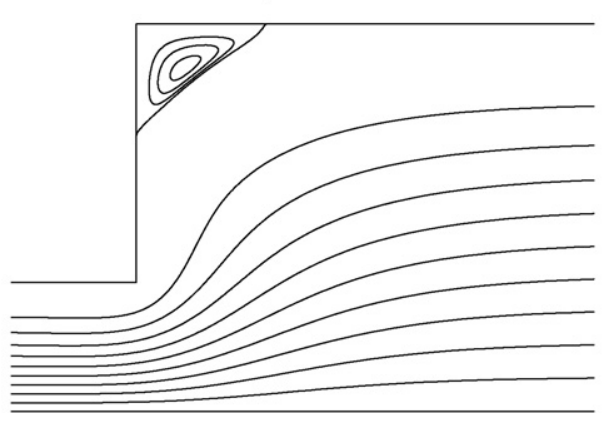

Fig. 13. Streamline patterns for the PTT model $\beta=1 / 9 \varepsilon=0.25$ (mesh M2A). Note: $\Delta \Psi_{\mathrm{R}}=0.1 \times 10^{-3}$ in recirculation region.

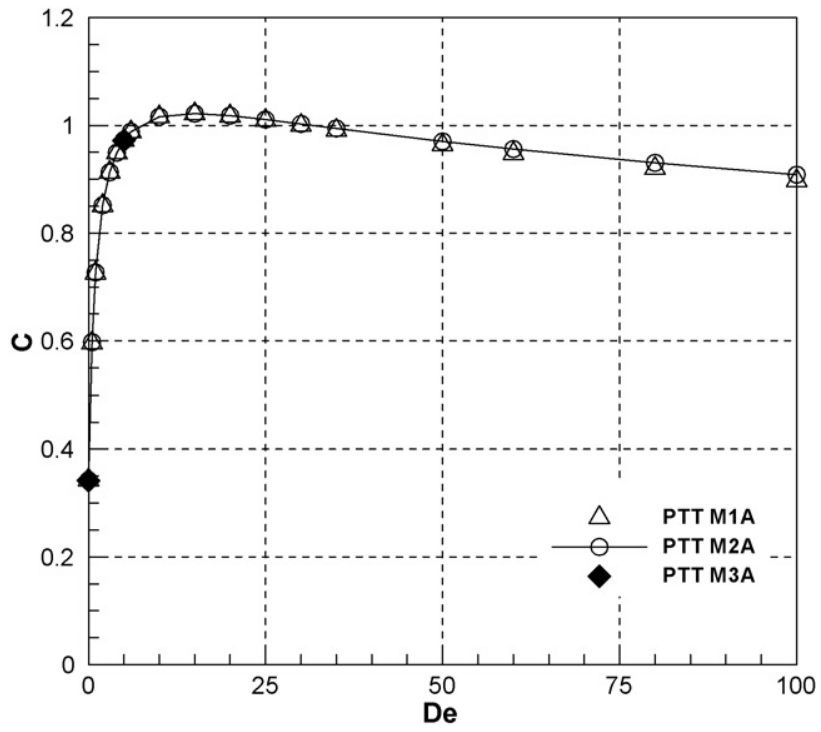

Fig. 14. Pressure drop across expansion with increasing elasticity for the PTT model. before gently decreasing at higher $\mathrm{De}$. This maximum pressure drop corresponds to the same level of elasticity at which the minimum $X_{\mathrm{R}}$ occurs in Fig. 11.

The variation of the streamwise velocity along the centreline of the expansion, in the vicinity of the expansion plane, is shown in Fig. 15. In Fig. 15(a), where we normalise with the bulk velocity in the inlet channel, the shear-thinning behaviour of the PTT model, as is well known, results in a flattening of the velocity profiles, varying the centreline velocity, and makes interpretation of the data difficult. In an attempt to remove the influence of shear-thinning, in Fig. 15(b) we normalise instead by the fullydeveloped centreline velocity for each fluid taken directly from our results in the upstream channel. This normalisation collapses the velocity in the upstream channel and allows us to observe, once again, the velocity overshoot immediately upstream of the expansion plane. Comparison of the simulated fully-developed centreline velocities $U_{\mathrm{c}}$ with the analytical solution of Cruz et al.[24] showed agreement to within $0.2 \% ; 1.387 / 1.385(D e=2)$, 1.378/1.377 $(D e=5), 1.387 / 1.385(D e=10)$ and 1.448/1.447 $(D e=100)$, (simulations/analytical). 


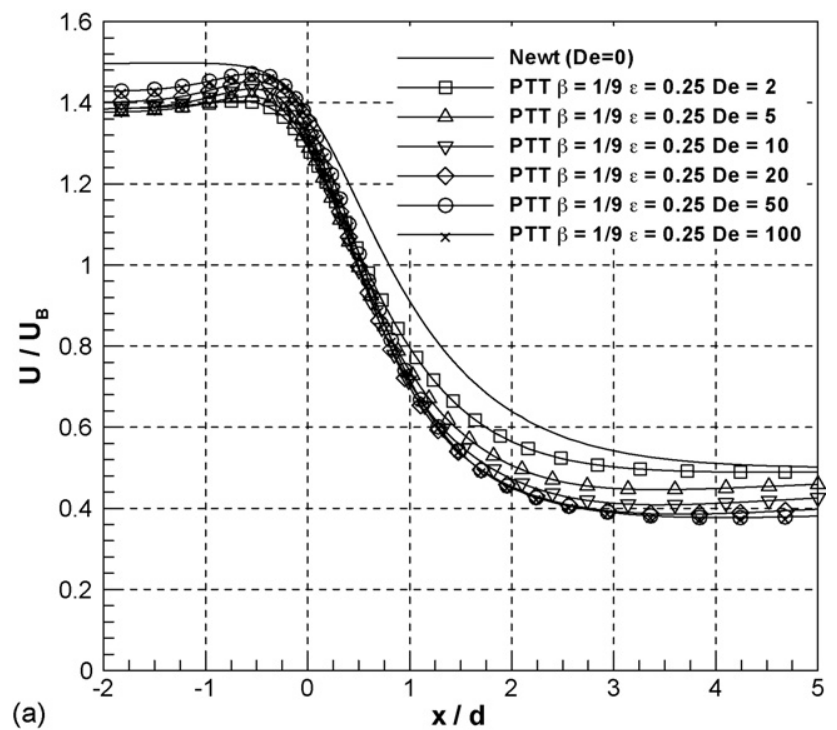

(a)

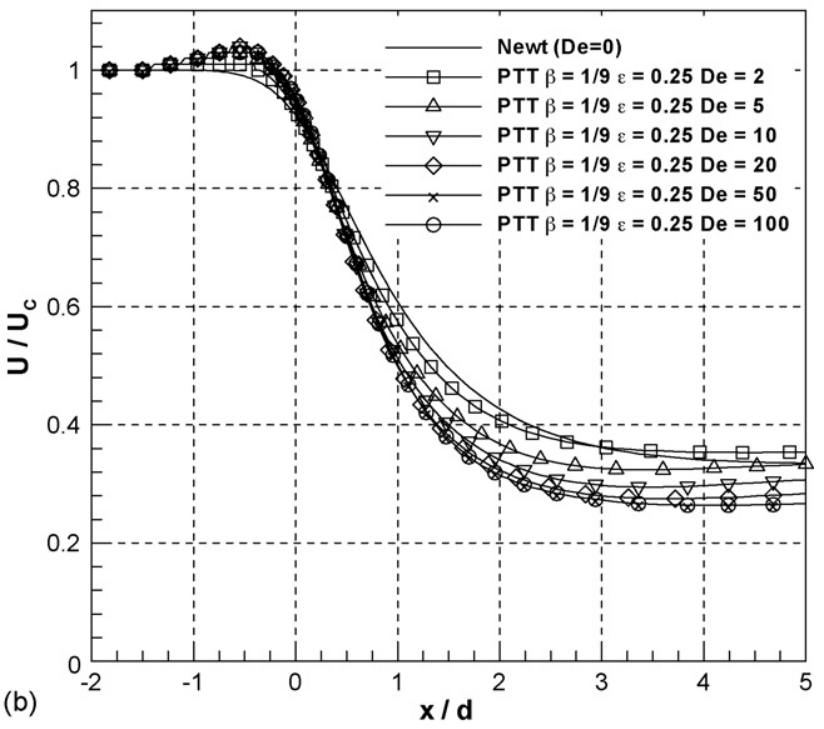

Fig. 15. Variation of streamwise velocity along centreline of expansion for the PTT model $(\beta=1 / 9, \varepsilon=0.25)$ Mesh M2A. To avoid overcrowding only limited data is shown. (a) Normalised by bulk velocity; (b) normalised by fully-developed centreline velocity in inlet duct.

Fig. 16 shows the asymptotic variation for the three stress components (polymer contribution only) along a streamwise line emanating from the expansion corner for the PTT model, $D e=5.0$ mesh M3A. The stresses grow to infinity at a rate proportional to $r^{-0.329}$, indicated by the lines of the same slope on the plot, in good agreement with the corresponding theoretical predictions for the PTT model [25].

Finally, in Fig. 17, we examine the influence of the $\varepsilon$ parameter on the reattachment length and intensity for the PTT model at $D e=1.0(\beta=1 / 9)$. Our rationale for this choice of $D e$ was that at this level of elasticity we could still obtain a converged solution for the limiting case (i.e. the Oldroyd-B model where $\varepsilon=0$ ). As can be seen, for this level of elasticity, the effect of $\varepsilon$ is rather small; $X_{\mathrm{R}}$ initially increases with increasing $\varepsilon$, reaching a peak at $\varepsilon=0.2$, an increase of about $5 \%$ compared to the Oldroyd-B value, before gradually decreasing. The effect on recirculation

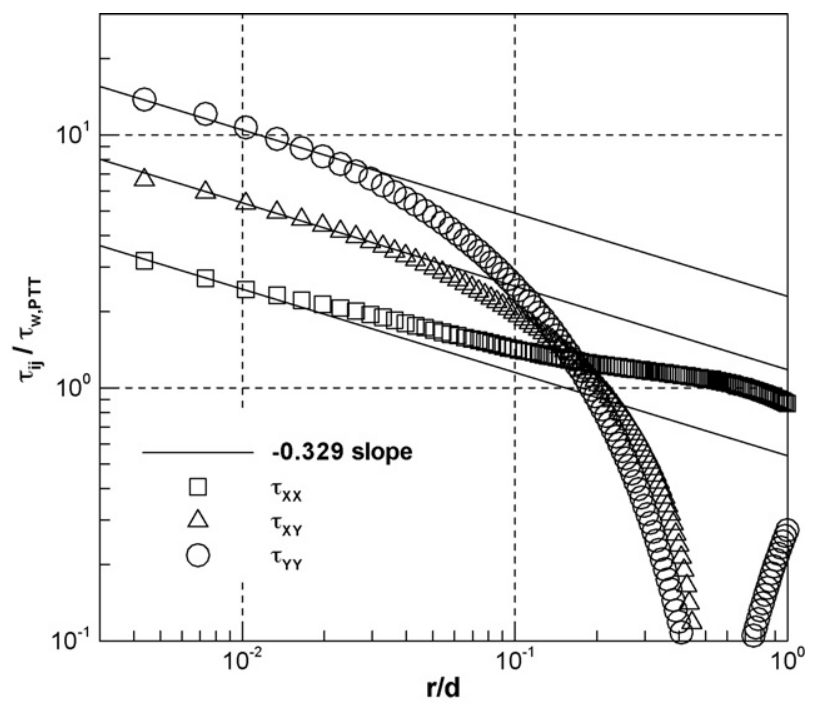

Fig. 16. Asymptotic variation of stresses along a streamwise line from expansion corner $(x, y=0, d)$ for the PTT model $(\beta=1 / 9, \varepsilon=0.25)$ De $=5.0$ using mesh M3A.

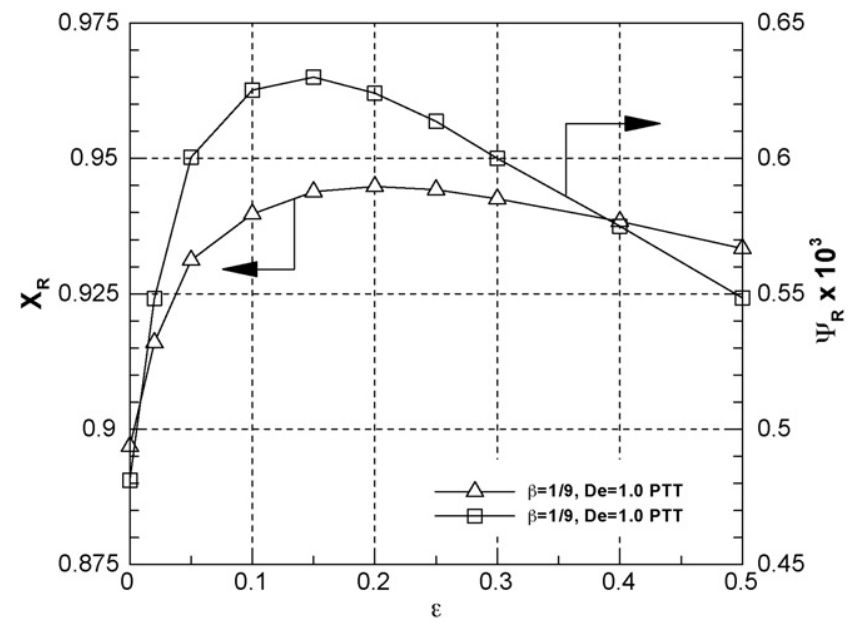

Fig. 17. Variation of recirculation length $X_{\mathrm{R}}$ and recirculation intensity $\Psi_{\mathrm{R}}$ with epsilon for the PTT model $(\beta=1 / 9, D e=1.0)$.

intensity is more pronounced: the peak value of $\Psi_{\mathrm{R}}$ occurs at a slightly lower value of $\varepsilon(=0.15)$, compared to the peak in $X_{\mathrm{R}}$, before once again slowly decreasing.

\section{Conclusions}

We have reported a systematic numerical investigation of the creeping flow of three model viscoelastic fluids, the UCM, Oldroyd-B and a simplified form of the PTT model, through a two-dimensional 1:3 planar sudden expansion. By utilising extremely fine meshes and mesh refinement centred around the corner singularity we have been able to estimate that the uncertainty in our simulations is very low. For the Newtonian, UCM and Oldroyd-B models the uncertainty in our estimate of the recirculation length downstream of the expansion is better than $0.15 \%$. For the PTT simulations we could not achieve converged solutions on the finest mesh for $D e>5$, for the simulations 
below this value the uncertainty of our results is again very low $(\sim 0.2 \%)$, above this value a noticeable (maximum difference $\sim 5 \%$ ) grid dependency is observable.

For the UCM and Oldroyd-B models the trends in the simulations are broadly similar and the following conclusions can be drawn; the maximum Deborah number that can be obtained is of order one, the initial effect of increasing elasticity is to reduce the recirculation length in comparison to the Newtonian fluid flow, this reduction in $X_{\mathrm{R}}$ plateaus at the higher Deborah numbers to a value roughly $17 \%$ less than the Newtonian value. So, in contrast to previous studies, we find that elasticity reduces but does not eliminate recirculation downstream of the expansion: concomitant changes are also observed in the intensity of the recirculation regions but these reductions are more significant $(\sim 60 \%)$. Estimation of the pressure drop across the expansion, expressed in terms of the Couette correction, shows an enhanced pressure drop for both models compared to the Newtonian fluid flow. This enhanced pressure drop increases with increasing $D e$. Velocity profiles along the centreline of the expansion reveal that a velocity overshoot occurs upstream of the expansion. The magnitude of this overshoot increases with $D e$. Although small this overshoot represents a convergence of the streamlines immediately upstream of the expansion plane: such converging streamlines can clearly be discerned in the 'zoomed' streamline plot for the Oldroyd-B simulation when $D e=1.4$ (Fig. 6). We believe that this convergence of the streamlines is responsible for the plateau observed in the recirculation length at high $D e$.

For the PTT model we were able to obtain iteratively converged solutions, using mesh M2A, up to much higher Deborah numbers $(\approx 100)$ and at low $D e$ observed similar effects to the UCM and Oldroyd-B simulations. At higher $D e$ an increase in the recirculation length in the streamwise direction was apparent but this was not matched by a corresponding increase in the recirculation intensity which continues to decrease albeit slowly. Profiles of velocity and stresses, when suitably normalised, were in qualitative agreement with the UCM and Oldroyd-B simulations. We suggest that the same mechanism, coupled with shear thinning, is responsible for this growth in recirculation length as for the levelling out observed in this quantity for the UCM and Oldroyd-B models.

\section{Acknowledgments}

RJP would like to thank the Engineering and Physical Sciences Research Council (EPSRC) who provided funding, via the Overseas Travel Grant programme (EP/D002052/1), that enabled his extended stay at the Universities of Minho, Porto and Beira Interior in summer 2005 where this work was initiated. RJP and MAA would also like to thank the British Council/Conselho de Reitores das Universidades Portuguesas for support through the 'Treaty of Windsor Programme' (LIS/992 (2005/06) U17 \& Acção Luso-Britânica n. B-17/05). MAA also acknowledges funding by Fundação para a Ciencia e a Tecnologia (FCT, Portugal) and FEDER under project POCI/EQU/59256/2004. PJO acknowledges partial funding by FCT and FEDER under project POCTI/EME/48665/2002. FTP and MAA acknowledge par- tial funding by FCT and FEDER under projects POCI/EQU/ 56342/2004 and POCI/EME/59338/2004).

\section{References}

[1] A.L. Halmos, D.V. Boger, Flow of viscoelastic polymer solutions through an abrupt 2-to-1 expansion, Trans. Soc. Rheol. 20 (1976) 253.

[2] A.L. Halmos, D.V. Boger, A. Cabelli, The behavior of a power-law fluid flowing through a sudden expansion. Part I. Numerical solution, AIChE J. 21 (1975) 540

[3] A.L. Halmos, D.V. Boger, A. Cabelli, The behavior of a power-law fluid flowing through a sudden expansion. Part II. Experimental verification, AIChE J. 21 (1975) 550.

[4] M.S. Darwish, J.R. Whiteman, M.J. Bevis, Numerical modelling of viscoelastic liquids using a finite-volume method, J. Non-Newt. Fluid Mech. 45 (1992) 311

[5] K.A. Missirlis, D. Assimacopoulos, E. Mitsoulis, A finite volume approach in the simulation of viscoelastic expansion flows, J. Non-Newt. Fluid Mech. 78 (1998) 91.

[6] M.A. Alves, P.J. Oliveira, F.T. Pinho, Benchmark solutions for the flow of Oldroyd-B and PTT fluids in planar contractions, J. Non-Newt. Fluid Mech. 110 (2003) 45.

[7] C.J. Freitas, Editorial policy statement on the control of numerical accuracy, ASME J. Fluids Eng. 115 (1993) 339.

[8] B.P. Leonard, J.E. Drummond, Why you should not use hybrid, power-law or related exponential schemes for convective modelling - there are much better alternatives, Int. J. Numer. Meth. Fluids 20 (6) (1995) 421.

[9] P. Townsend, K. Walters, Expansion flows of non-Newtonian liquids, Chem. Eng. Sci. 49 (1994) 749

[10] N. Phan-Thien, R.I. Tanner, A new constitutive equation derived from network theory, J. Non-Newt. Fluid Mech. 2 (1977) 353.

[11] A. Baloch, P. Townsend, M.F. Webster, On vortex development in viscoelastic expansion and contraction flows, J. Non-Newt. Fluid Mech. 65 (1996) 133.

[12] P.J. Oliveira, Asymmetric flows of viscoelastic fluids in symmetric planar expansion geometries, J. Non-Newt. Fluid Mech. 114 (2003) 33.

[13] W. Cherdron, F. Durst, J.H. Whitelaw, Asymmetric flows and instabilities in symmetric ducts with sudden expansions, J. Fluid Mech. 84 (1978) 13.

[14] R.M. Fearn, T. Mullin, K.A. Cliffe, Non-linear flow phenomena in a symmetric sudden expansion, J. Fluid Mech. 1990 (1990) 595.

[15] F. Durst, A. Melling, J.H. Whitelaw, Low Reynolds number flow over a plane sudden expansion, J. Fluid Mech. 64 (1974) 111.

[16] M.D. Chilcott, J.M. Rallison, Creeping flow of dilute polymer solutions past cylinders and spheres, J. Non-Newt. Fluid Mech. 29 (1988) 381

[17] J.G. Oldroyd, On the formulation of rheological equations of state, Proc. Roy. Soc. London A 200 (1950) 523.

[18] R.G. Owens, T.N. Phillips, Computational Rheology, Imperial College Press, London, 2002.

[19] P.J. Oliveira, F.T. Pinho, G.A. Pinto, Numerical simulation of non-linear elastic flows with a general collocated finite-volume method, J. Non-Newt. Fluid Mech. 79 (1998) 1.

[20] P.J. Oliveira, On the numerical implementation of non-linear viscoelastic models in a finite-volume method, Numer. Heat Transfer, Part B 40 (2001) 283.

[21] M.A. Alves, P.J. Oliveira, F.T. Pinho, A Convergent and Universally Bounded Interpolation Scheme for the Treatment of Advection, Int. J. Num. Methods Fluids 41 (2004) 47.

[22] P.H. Gaskell, A.K.C. Lau, Curvature-compensated convective transport: Smart, a new boundedness preserving transport algortithm, Int. J. Numer. Meth. Fluids 8 (1988) 617.

[23] E.J. Hinch, The flow of an Oldroyd-B fluid around a sharp corner, J. NonNewt. Fluid Mech. 50 (1993) 161.

[24] D.O. Cruz, F.T. Pinho, P.J. Oliveira, Analytical solution for fully developed laminar flow of some viscoelastic liquids with a Newtonian solvent contribution, J. Non-Newt. Fluid Mech. 132 (2005) 28.

[25] M. Renardy, Re-entrant corner behaviour of the PTT fluid, J. Non-Newt. Fluid Mech. 69 (1997) 99. 